\title{
Evaluating Computer Capabilities in a Primary Care Practice-Based Research Network
}

\author{
Adolfo J. Ariza, MD \\ Helen J. Binns, MD, MPH \\ Katherine Kaufer Christoffel, MD, \\ $\mathrm{MPH}$, for the Pediatric Practice \\ Research Group \\ Mary Ann and J. Milburn Smith Child \\ Health Research Program, Children's Memo- \\ rial Hospital, Feinberg School of Medicine, \\ Northwestern University, Chicago, Ill
}

\begin{abstract}
PURPOSE We wanted to assess computer capabilities in a primary care practicebased research network and to understand how receptive the practices were to new ideas for automation of practice activities and research.

METHOD This study was conducted among members of the Pediatric Practice Research Group (PPRG). A survey to assess computer capabilities was developed to explore hardware types, software programs, Internet connectivity and data transmission; views on privacy and security; and receptivity to future electronic data collection approaches.

RESULTS Of the 40 PPRG practices participating in the study during the autumn of 2001, all used IBM-compatible systems. Of these, $45 \%$ used stand-alone desktops, 40\% had networked desktops, and approximately 15\% used laptops and minicomputers. A variety of software packages were used, with most practices (82\%) having software for some aspect of patient care documentation, patient accounting (90\%), business support (60\%), and management reports and analysis (97\%). The main obstacles to expanding use of computers in patient care were insufficient staff training (63\%) and privacy concerns (82\%). If provided with training and support, most practices indicated they were willing to consider an array of electronic data collection options for practice-based research activities.
\end{abstract}

CONCLUSIONS There is wide variability in hardware and software use in the pediatric practice setting. Implementing electronic data collection in the PPRG would require a substantial start-up effort and ongoing training and support at the practice site.

Ann Fam Med 2004;2:418-420. DOI: 10.1370/afm.114.

\section{INTRODUCTION}

$\mathrm{P}$ ractice-based networks study issues important to primary care delivery. ${ }^{1,2}$ To guide improvements in practice-based research methods, it will be important to understand current computer systems. ${ }^{3}$ In 2001 the Pediatric Practice Research Group (PPRG), an experienced, regional network, ${ }^{1,2}$ undertook an assessment of computer capabilities in a goal to foster data system developments. This article presents our findings.

\section{METHODS}

A 14-page questionnaire was developed to assess systematically the electronic data capacity and communications in practice settings. Some questions were adapted from survey questionnaires previously developed by Children's Memorial Hospital and the American Academy of Pediatrics Pediatric Research in Office Setting network. This survey was designed to assess the practices and their computer systems, including software 


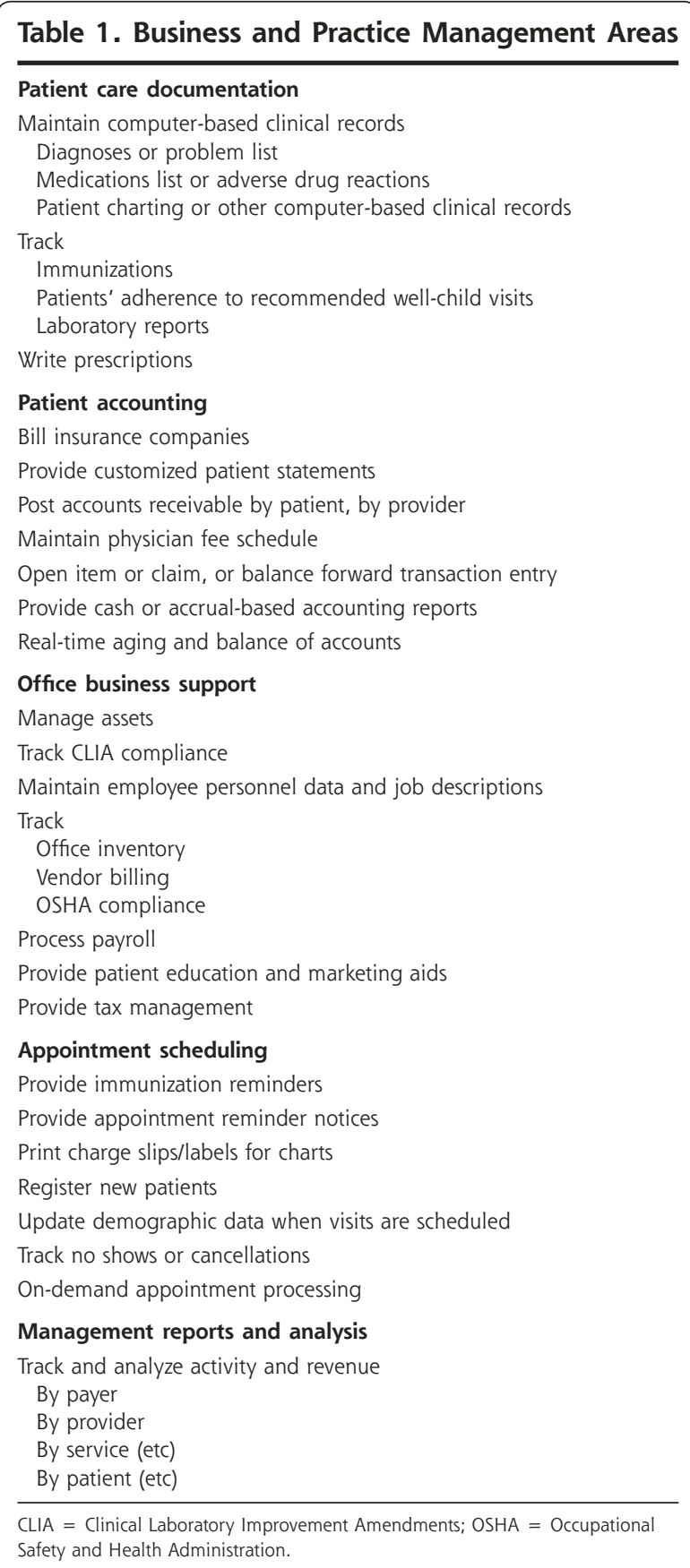

hardware, privacy, security and confidentiality, and future computer uses. Questions were forced choice or numeric response.

Software topics sought information on software uses for (1) patient care documentation, (2) patient accounting, (3) office business support, (4) appointment scheduling, and (5) management reports and analysis. The elements assessed in each area are displayed in Table 1. A practice was considered as using software for an area if one or more specific uses were identified. Hardware topics included questions regarding type and number of computers, Internet connectivity, and data transmission capabilities. Confidentiality topics focused on handling paper and electronic records. Practice acceptance and preparedness evaluated readiness for new data collection approaches and obstacles to expanded computer use.

\section{RESULTS}

Forty practices ( $83 \%$ of invited) representing 96 offices and 194 pediatricians returned a completed questionnaire. For each practice, a key administrator and physician completed the questionnaire.

\section{Computer Capabilities}

All practices used IBM-compatible computer systems, and $90 \%$ owned their hardware. There was wide variability in types of hardware used; $45 \%$ used stand-alone desktop microcomputers and $40 \%$ used networked microcomputers; $22 \%$ used notebook or laptop microcomputers, and $7 \%$ used minicomputers.

Most practices used software for management reports and analysis (97\%), patient accounting (87\%), and patient care documentation (60\%). Approximately one half $(60 \%)$ used software for business support, and $25 \%$ had appointment scheduling software. Among the 33 practices using software for patient care documentation, 30\% used Medical Manager, (Medical Manger PCN, Inc, Fairfield, NJ), and the rest used any of 28 other software programs. Table 2 displays practice use of software for each aspect of patient care management.

Internet connections were available at $87 \%$ of practices, but the number of connected terminals varied: $20 \%$ had all computer terminals connected, 50\% had some, and $15 \%$ had 1 . Among the 35 practices with an Internet connection, $55 \%$ used it for electronic mail, $25 \%$ for their home page, and $65 \%$ to access the Web. Thirty-three percent used the Internet for data submission. The following were common obstacles to expanding Internet use: not enough computers for business and Internet use $(53 \%)$, insufficient staff training or time $(50 \%)$, and privacy concerns $(50 \%)$.

Most respondents $(82 \%)$ had concerns about patient confidentiality ( $27 \%$ a lot, $27 \%$ moderate, $32 \%$ some, $14 \%$ minor), primarily regarding security of electronic records. Twenty-eight percent perceived themselves as having insufficient security for patient confidentiality with electronic information.

Respondents were open to increased use of computers for research data collection. Most were willing to consider handheld touch-screen devices not linked to the practice system $(63 \%)$, downloading questionnaires from the Web (66\%), and Web-based questionnaires (58\%). 


\begin{tabular}{|lc|}
\hline \multicolumn{2}{l}{ Table 2. Use of Software for Patient Care } \\
Documentation \\
\hline Computer-Based Clinical Records & Percent Using \\
\hline Diagnoses or problem list & 38 \\
Medication list, including adverse drug reactions & 5 \\
All patient charting & 0 \\
Other computer-based clinical records (specify) & 8 \\
Track immunization & 25 \\
Track patients' adherence to recommended & 20 \\
$\quad$ well-child visits & 15 \\
Track laboratory reports & 13 \\
Write prescriptions & 13 \\
Provide information on drug interactions & 10 \\
Provide information on chronic disease & \\
management & 10 \\
Provide telephone triage protocols for office staff & 0 \\
Accept dictation directly into patients' computer & \\
records via voice recognition & 18 \\
Internet access (eg, person on call, emergency & \\
$\quad$ department staff, etc) & 38 \\
Diagnoses or problem list & \\
\hline
\end{tabular}

There were many obstacles to expanding computer use: insufficient staff training and time $(61 \%)$, hardware and software limitations $(55 \%)$, and costs $(40 \%)$.

\section{DISCUSSION}

Our study provides new information relevant to primary care practice-based research network computer capabilities.

"Our network practices use PCs, not Macs." Electronic data collection must take into account the compatibility of operating systems and the variety of experiences with computers, including differences in training.

A great variability exists in the software used. Business and practice management tasks that are computerized vary between sites. Software variations appeared to relate more to which tasks were computerized at a particular practice; there was some consistency in software choices for particular practice management and patient care areas.

Computers were not consistently connected to the Internet. This finding seems to relate to insufficient hardware, staff time, training issues, and concerns about data privacy. Data collection approaches cannot, at this time, assume that office computers can connect to the Web. It also indicates that there are training needs-and opportunities - related to Internet use, and that practices must be assured excellent data security measures before they engage in data transfer over the Internet.

Clinicians and their practices were open to expand- ing computer use. To facilitate such a move, obstacles that have kept computer use low must be addressed, including limitations in staff time, current availability of hardware and software, and training. Studies that use computer technology must include a budget to overcome these limitations.

"Practices in our network are all different." This finding affects all aspects of practice operation. As a result, current systems do not allow for standardized computer data collection for research purposes.

\section{Limitations}

This study was conducted in a single, regional pediatric research network among diverse practices, so generalization from the findings presented must be done with caution. The data for this study was collected in 2000-2001, and the technology landscape is changing rapidly.

\section{CONCLUSIONS}

All surveyed practices in our network used PCs. A minority of computers were connected to the Internet. PPRG electronic data collection approaches must account for the variety of software, staffing, and computer training needs found. For Internet data collection in practices such as ours, a substantial start-up effort will be required. These data can guide planning by networks with similar practice structures.

To read or post commentaries in response to this article, see it online at http://www.annfammed.org/cgi/content/full/2/5/418.

Key words: Primary health care; practice-based research; automatic data processing; computer communication networks

Submitted May 1, 2003; submitted, revised, November 3, 2003; accepted December 3, 2003.

Portions of this paper were presented at the Pediatric Academic Societies' Annual Meeting, Baltimore, Md, May 4, 2002.

Funding support: Agency for Healthcare Research and Quality, Grant No. P2OHS11248

\section{References}

1. Christoffel KK, Binns HJ, Stockman JA, et al and the Pediatric Practice Research Group. Practice-based research: opportunities and obstacles. Pediatrics. 1988;82:399-406.

2. LeBailly S, Ariza A, Bayldon B, Binns HJ. The origin and evolution of a regional pediatric practice-based research network: practical and methodological lessons from the Pediatric Practice Research Group. Curr Probl in Pediatr Adolesc Health Care. 2003;33:101-140.

3. Agency for Healthcare Research and Quality. Primary Care PracticeBased Networks Fact Sheet. Publication 01-P020. June 2001. Available at: http:www.ahrq.gov/research/pbrnfact.htm. 